\title{
PERÍODOS CONDICIONALES GRIEGOS: UN ANÁLISIS LINGÜÍSTICO
}

The author analyses Greek conditional periods using texts by Sophocles, Thucydides and Plato, and applies a structural method with a functional approach, in an attempt to assign constant function, value and meaning to each formal expression. After rejecting the traditional names used to denote conditionals, the author draws up a table of nine levels of theoretical combinations of which only seven have been recorded in the examples studied.

\section{INTRODUCCIÓN}

1. En un artículo publicado en $1989^{\prime}$ exponíamos el estado actual de los estudios relativos a los períodos condicionales griegos. En él llegábamos a la conclusión de que era necesario hacer un análisis nuevo en el que se delimitara el objeto, se aplicara un criterio homogéneo a lo largo de la investigación y se llegara a un resultado claro y coherente. Hemos entendido que existía ya un precedente en la sintaxis latina, el estudio de $\mathrm{H}$. Vairel ${ }^{2}$ sobre el elemento subordinado de un período condicional. En nuestro caso hemos considerado que los períodos condicionales griegos, como en otras lenguas, han de ser estudiados en su conjunto, tanto el elemento subordinado como el elemento principal. Respecto al trabajo de Vairel hay, por consiguiente, un avance.

2. Partiendo del principio de que en un período condicional se establece entre sus dos miembros, prótasis y apódosis, una relación de condición a consecuencia, entendida como de condicionante a condicionado ${ }^{3}$, la función de la conjunción subordinada condicional no consiste en poner en relación los contenidos de los elementos subordinado y

' «Periodos condicionales griegos. Estudio crítico», EC 96, 1989, pp. 75-95.

2 "Un modèle d'analyse linguistique des conditionnelles: Latin si di sunt, si di sint, si di essent", BSL 76, 1, 1981, pp. 275-326.

${ }^{3}$ H. Vairel, art. cit., p. 276. 
principal, como se interpreta habitualmente, sino que la función de la conjunción subordinada es poner en relación dos actos de discurso: uno, el de suponer lo que se dice en la prótasis; dos, el de enunciar lo que se dice en la apódosis. Esta diferencia de matiz es esencial para comprender la significación que tiene cada uno de los diversos períodos condicionales. Así, en la expresión «si llueve, las calles se mojan» la función de la conjunción "si» no es relacionar «llueve» con "se mojan las calles», sino relacionar el acto de suponer que, de llover, las calles se van a mojar.

3. En griego los dos miembros, prótasis y apódosis, reciben otras denominaciones como primer y segundo términos del período, subordinado y principal, condicionante y condicionado, etc. Cada miembro aparece bajo expresiones diferentes y su combinación ha dado lugar a las tradicionales definiciones de condicionales "reales», "eventuales», «potenciales» o "posibles», «irreales», «iterativas de presente», «iterativas de pasado", "mixtas», etc. Dado que en estas denominaciones no se ha aplicado constantemente el mismo criterio, ni siquiera se está de acuerdo con ellas, está justificado que se intente un nuevo análisis con las siguientes características:

a) Estudio de cada una de las expresiones bajo las que aparecen el condicionante y el condicionado, sus combinaciones posibles y las que se realizan en la práctica.

b) Consideración del objeto de estudio desde una perspectiva exclusivamente lingüística, excluyendo aquellas apreciaciones filosóficas o contextuales que difuminen la objetividad en la interpretación.

c) Enfoque estructural en cuanto que cada expresión ha de cumplir una función en su oposición respectiva, distinguiendo un término marcado y otro no marcado, caracterizado aquél mediante una marca formal que implique la noción básica sobre la que se establece la oposición. De este modo, la expresión marcada tendrá un valor positivo y significará algo de lo que carecerá la expresión no marcada; ésta tendrá valor negativo.

d) Estas bases metodológicas son aplicadas a un número de textos con el fin de que se pueda comprobar la validez o no del análisis sugerido. No se considera imprescindible una selección exhaustiva de autores u obras, sino sólo una muestra simbólica. Concretamente se han seleccionado los ejemplos que aparecen en Antígona de Sófocles, Protágoras de Platón y el fragmento II 1 - II 46 de Tucídides.

e) Agrupados los ejemplos según la forma verbal de la prótasis y de la apódosis, se observa la frecuencia de cada una de las combinacio- 
nes posibles en cada autor y se hace una aproximación a la relación que pueda haber entre los tipos de períodos condicionales resultantes y el tipo de lenguaje analizado (dramático, filosófico e histórico), así como si corresponde a parte dialogada o no. Aquellos períodos condicionales cuya forma verbal aparece en modo no personal, infinitivo o participio, son eliminados del análisis por entender que dependen a su vez de alguna expresión anterior o sobreentendida; también se elimina el texto que contenga un optativo oblicuo. Por otra parte, son eliminados aquellos períodos que no son sólo condicionales, sino que en ellos interviene algún elemento comparativo o relativo. Así pues, son analizados los períodos condicionales introducidos por las conjunciones subordinadas $\varepsilon \dot{l}$, źáv, ந̆v o ăv seguidas de forma verbal personal.

f) Este estudio permitirá una nueva definición de las condicionales y abrirá un nuevo camino en el análisis estilístico.

\section{Hipótesis de tRabajo}

4. Prótasis. El primer término de un período condicional presenta en los textos analizados las expresiones siguientes:
a) $\varepsilon l+$ ind.,
b) záv / $\not u v / a ̈ v+s u b j$. ,
c) $\varepsilon l+$ opt.

Estas tres variantes pueden ser reducidas a dos en un primer paso: una, prótasis en indicativo; dos, prótasis en subjuntivo u optativo. En un segundo paso es necesario situar adecuadamente una cuarta expresión, la formada por $\varepsilon l+$ fut. ind. En efecto, la prótasis en fut. de ind. ha sido considerada por los investigadores como una condicional real más, en consonancia con el valor "real» asignado generalmente al modo indicativo. Pero resulta que su enunciado es una suposición de algo que por su propio significado temporal de futuro no tiene aún existencia $\mathrm{y}$, por tanto, no puede ser «real». Otros han relacionado su proyección futura con la eventualidad atribuida al subjuntivo, pero matizando algún detalle que los diferenciara. La práctica, sin embargo, nos demuestra que, en efecto, la prótasis en futuro de indicativo proyecta el enunciado de la suposición hacia un momento posterior al acto lingüístico, lo que la diferencia de las otras expresiones en indicativo. Por otro lado, los períodos condicionales de prótasis múltiple con presencia simultánea de expresiones en futuro de indicativo y en subjuntivo tienen sus apódosis expresadas en imperativo o en futuro de indicativo, coincidencia que no 
se registra cuando se trata de prótasis múltiple de futuro y otras formas temporales del indicativo (presente y pasado). Se puede afirmar, por tanto, que la protásis expresada con $\varepsilon l+$ fut. ind. desempeña una función semejante a la que realiza la prótasis expresada con źáv + subj., por lo que es conveniente incluir la condicional expresada en fut. de ind., junto a las expresadas en subjuntivo u optativo, como se establecía al comienzo de este párrafo.

5. La prótasis en indicativo (no futuro) funciona como término no marcado en cuanto que esa expresión no está caracterizada formalmente en la oposición modal indicativo / subjuntivo (fut. ind.) - optativo. En un período condicional, en efecto, el modo indicativo no añade matiz subjetivo alguno al enunciado de la suposición. A este respecto conviene recordar que el indicativo no es exactamente el modo de la uobjetividad» o de la "realidad», como es habitualmente definido, sino que, negativamente, es el modo que menos subjetividad expresa ${ }^{4}$.

6. La prótasis en subjuntivo, futuro de indicativo y optativo funciona como término marcado, al estar caracterizada cada una de ellas por la vocal de unión alargada, la sigma y la iota respectivamente, en términos generales ${ }^{5}$. En un período condicional sí añade al enunciado de la suposición una aportación subjetiva que más adelante concretaremos. Como se ha indicado en $\S 4$, la diferencia que cabe establecer entre las expresiones en futuro de indicativo y en subjuntivo se puede definir no tanto como una diferencia de significación, pues semánticamente no se aprecia, cuanto como una doble realización en el plano del habla sin diferencia significativa en el plano de la lengua. De hecho, la expresión en futuro de indicativo es registrada en una proporción considerablemente menor que la registrada en subjuntivo, por lo que cabría preguntarse si la explicación del uso de fut. de ind. en una prótasis no obedece a una cierta atracción modal de aquellas apódosis expresadas en futuro de indicativo.

7. Asi pues, las prótasis de un período condicional pueden distribuirse así:

a) $\varepsilon l+$ indicativo (no futuro).

b) $\varepsilon a ́ v+$ subjuntivo $/ \varepsilon l+$ futuro de indicativo $/ \varepsilon l+$ optativo.

${ }^{4}$ Cf. Vairel, art. cit., p. 290.

' Es obvia la marca de estas expresiones en circunstancias particulares como puede ser la del subjuntivo del verbo atemático en Homero o la del futuro no sigmático. 
Ello quiere decir que el término marcado de una prótasis se realiza en griego con tres variantes. Habrá que explicar, pues, qué significa el término marcado y qué diferencias existen, si las hay, entre cada una de estas expresiones.

8. La noción básica del término marcado consiste, como se recogía en $\S 6$, en una segunda apreciación subjetiva en el enunciado de la prótasis. Recordemos que todo período condicional es en sí mismo un acto lingüístico subjetivo (primera aportación): el hablante formula una suposición en la que dado un caso (supuesto), se produce necesariamente una consecuencia. Pues bien, puede haber un segundo matiz subjetivo, que denominaremos toma de posición del hablante, cuando éste añade al enunciar una suposición su proyección temporal hacia un momento anterior, simultáneo o posterior con respecto al acto lingüístico. En efecto, mientras que una suposición en indicativo no futuro se limita simplemente a su enunciado, con la única referencia, propia de la forma verbal, de su actualidad o de su no-actualidad, la suposición expresada en subjuntivo, futuro de indicativo y optativo añade al enunciado una proyección temporal. Las tres expresiones poseen la noción básica del término marcado (segundo acto subjetivo consistente en proyectar temporalmente el enunciado de la suposición), pero la realizan en diferente grado. Así, la prótasis en optativo proyecta el enunciado de la suposición hacia un momento "no necesariamente posterior» al acto lingüístico. La prótasis en subjuntivo o en futuro de indicativo proyecta el enunciado de la suposición hacia un momento «necesariamente posterior» al acto lingüístico. Por ello la noción básica es realizada en dos grados: a) Grado uno, representado por el optativo, significa que el momento respecto al acto lingǘstico no se especifica necesariamente, puede ser anterior (no registrado en nuestro análisis), simultáneo o posterior; dentro del término marcado es el que menos definición posee. b) Grado dos, representado por el subjuntivo y el futuro de indicativo, significa que el momento hacia el que se proyecta la suposición respecto al acto linguístico está precisado, es necesariamente posterior; dentro del término marcado es el que está más definido.

9. Las expresiones en indicativo no futuro constituyen, por tanto, el término no marcado y carecen de la noción básica, limitándose al enunciado de la suposición sin otra connotación subjetiva. Ésta podría ser la razón por la que se han considerado las expresiones en indicativo no futuro [y futuro] como «reales»; pero debe quedar claro que todo período condicional es enunciado como algo que se supone en su realidad, independientemente de la forma verbal en la que esté expresado. 
De ahí que consideremos inadecuada y errónea su denominación como «reales».

10. Veamos algunos ejemplos:

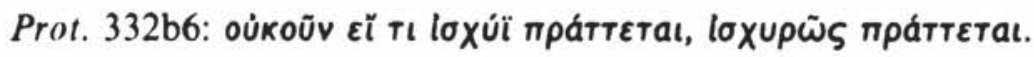

Tiene la prótasis en pres. de ind., por lo que sólo cabe entender el acto subjetivo de la suposición: "si algo se hace con fuerza, se hace fuertemente». La forma verbal de la prótasis indica que la suposición está planteada en el mismo momento que el acto lingüístico; el hablante se limita a enunciar el contenido de la suposición y su consecuencia.

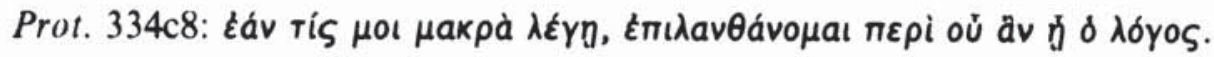

Tiene su prótasis en subjuntivo, por lo que se entiende que además de enunciar el contenido de la prótasis, éste es proyectado hacia un momento posterior al acto lingüístico: «en el caso de que alguien me hable extensamente [a partir de ahora, con posterioridad a este momento], me olvido..."

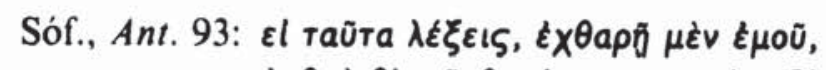

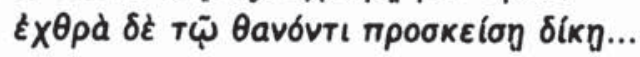

Su prótasis está en fut. de ind., por lo que, al igual que en el ejemplo anterior, el contenido del elemento condicionante es proyectado hacia un momento posterior al acto lingüístico: «en el caso de que hables asísi vas a hablar así [no existe en castellano una expresión equivalente a la griega], serás aborrecida por mí».

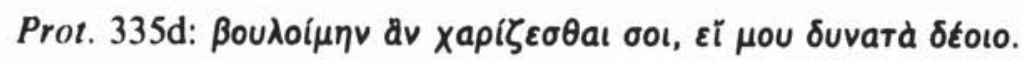

Tiene su prótasis en optativo, por lo que cabe interpretar que el hablante añade al contenido de la suposición una proyección temporal no necesariamente posterior, pudiéndose interpretar como simultáneo o posterior al acto lingüístico: «si tú me pidieses [ahora / más tarde] algo posible, querría hacerte el favor». 
11. Se puede representar la prótasis en el cuadro siguiente:

\begin{tabular}{|c|c|c|c|c|c|c|}
\hline \multicolumn{7}{|c|}{ Prótasis } \\
\hline Expresión & Marca & $\begin{array}{c}\text { Noción } \\
\text { básica }\end{array}$ & Significación & Función & Valor & $\begin{array}{c}\text { Represen- } \\
\text { tación }\end{array}$ \\
\hline $\begin{array}{c}\varepsilon l+\text { ind. } \\
\text { (no futuro) }\end{array}$ & $\theta$ & $\begin{array}{l}\text { no hay toma } \\
\text { de posición } \\
\text { del hablante }\end{array}$ & $\begin{array}{l}\text { sin proyec. } \\
\text { temporal } \\
\text { añadida }\end{array}$ & $\begin{array}{l}\text { término } \\
\text { no } \\
\text { marcado }\end{array}$ & negativo & $P-$ \\
\hline$\varepsilon l+$ opt. & $-1-$ & $\begin{array}{l}\text { hay toma } \\
\text { de posición } \\
\text { del hablante }\end{array}$ & $\begin{array}{l}\text { proyec. temp.: } \\
\text { no necesaria- } \\
\text { mente posterior }\end{array}$ & $\begin{array}{l}\text { término } \\
\text { marcado }\end{array}$ & $\begin{array}{l}\text { positivo } \\
\text { grado } 1\end{array}$ & $P+1$ \\
\hline $\begin{array}{c}\varepsilon a ́ v / \tilde{\eta} v / \\
a ̈ v / \varepsilon l+ \\
\text { subj. } \\
\varepsilon l+\text { fut. } \\
\text { de ind. }\end{array}$ & $\begin{array}{l}\text { vocal } \\
\text { unión } \\
\text { alar- } \\
\text { gada } \\
-\sigma-\end{array}$ & $\begin{array}{l}\text { hay } \\
\text { toma } \\
\text { de } \\
\text { posición } \\
\text { del } \\
\text { hablante }\end{array}$ & $\begin{array}{l}\text { proyección } \\
\text { temporal: } \\
\text { necesariamente } \\
\text { posterior }\end{array}$ & $\begin{array}{l}\text { término } \\
\text { marcado }\end{array}$ & $\begin{array}{l}\text { positivo } \\
\text { grado } 2\end{array}$ & $P+2$ \\
\hline
\end{tabular}

12. Apódosis. El segundo término de un periodo condicional es considerado como la consecuencia o enunciado condicionado y equivale a un contenido gramaticalmente independiente. En los textos analizados se han registrado las siguientes expresiones:

a) Expresiones sin partícula modal åv (ind., imperat., subj. y opt.).

b) Expresiones con partícula modal ăv (ăv + ind. y ăv + opt.).

Las expresiones que llevan la partícula modifican el sentido originario del modo verbal añadiendo una significación de la que ese modo por sí solo carece. Numerosos estudios han asignado al optativo sin partícula una significación desiderativa, mientras que se da significación potencial al optativo acompañado de ăv. Por su parte, el indicativo ha sido considerado como el modo de la objetividad o realidad, con significación lógica o simplemente declarativa ${ }^{6}$; en cambio, cuando va con ăv adquiere significación de irrealidad. Por tanto, las expresiones verbales que en la apódosis de un período condicional lleven la partícula modal funcionarán como término marcado, por cuanto que la presencia de la partícula modifica claramente la significación propia del modo verbal

- Cf. A. García Calvo, «Preparación a un estudio orgánico de los modos verbales sobre el ejemplo del griego antiguo", EMERITA 28, 1960, pp. 1-47. 
usado. Dicha partícula será el elemento portador de la noción básica, como en la prótasis lo era la vocal de unión alargada, la sigma o la iota de subj., fut. y opt. respectivamente. El término no marcado en la oposición será aquél que no tenga ăv.

13. En la apódosis la noción básica del término marcado significa, como en la prótasis, una toma de posición del hablante al enunciar el elemento condicionado por cuanto añade una consideración subjetiva, aquella que está implícita en el uso de ăv con optativo e indicativo. En efecto, entendemos que la expresión verbal acompañada de la partícula añade a la significación propia del modo (ind. y opt.) una apreciación subjetiva (toma de posición) que se concreta en un grado de seguridad personal acerca de la realización del período condicional en su conjunto. Por tanto, ello quiere decir: a) el indicativo y el optativo con partícula modal significan algo diferente del sentido originario de esos modos; b) su significación afecta no sólo a la apódosis en sí, sino al conjunto formado por los dos miembros del período condicional (recordemos que es una expresión condicionada); y c) al ser dos las expresiones que tienen la marca portadora de la noción básica cada una de ellas poseerá esa noción en un grado distinto.

14. Así, el opt. + äv funcionará como término marcado (presencia de la partícula), tendrá valor positivo y significará que el hablante toma posición ante el enunciado del condicionado al añadir que él no está seguro de la realización del período condicional. Obsérvese que insistimos en la realización de los dos miembros, a diferencia de las teorías tradicionales, pues entendemos que en todo período condicional el nexo que une condicionante y condicionado es siempre necesario, es decir, es siempre supuesto como real, cierto o seguro. Esta toma de posición expresada por el optativo y que se concreta en su no seguridad, constituye el grado uno en la posesión de la noción básica.

15. El ind. + ăv funcionará como término marcado (presencia de la partícula), tendrá valor positivo y significará que el hablante toma posición ante el enunciado del condicionado al añadir que él no sólo no está seguro de la realización del período (grado uno), sino que además está seguro de su no realización (grado dos). Como en el caso anterior, se debe insistir en que el grado de seguridad no afecta a la apódosis sólo, como se ha sostenido hasta ahora, sino que afecta al conjunto del periodo condicional.

16. El término no marcado en la apódosis está representado por aquellas expresiones verbales que carecen de ăv: indicativo, imperativo, 
subjuntivo y optativo. Dado que la marca portadora de la noción básica está ausente, su valor es negativo y su significación se limita al enunciado del elemento condicionado, sin otro matiz que el implicado en cada modo verbal: menor subjetividad, orden, exhortación, deseo.

17. El siguiente cuadro esquematiza la apódosis:

\begin{tabular}{|c|c|c|c|c|c|c|}
\hline \multicolumn{7}{|c|}{ Apódosis } \\
\hline Expresión & Marca & $\begin{array}{l}\text { Noción } \\
\text { hásica }\end{array}$ & Significación & Función & Vulor & $\begin{array}{c}\text { Represen- } \\
\text { tación }\end{array}$ \\
\hline $\begin{array}{l}\text { indicativo, } \\
\text { imperativo, } \\
\text { subj., opt. }\end{array}$ & $\varnothing$ & $\begin{array}{l}\text { no hay toma } \\
\text { de posición } \\
\text { del hablante. }\end{array}$ & $\begin{array}{l}\text { sin añadir } \\
\text { matiz subjetivo } \\
\text { a forma verbal }\end{array}$ & $\begin{array}{l}\text { término } \\
\text { no } \\
\text { marcado }\end{array}$ & negativo & A - \\
\hline ăv + opt. & ăv & $\begin{array}{l}\text { hay toma } \\
\text { de posición } \\
\text { del hablante }\end{array}$ & $\begin{array}{l}\text { no seguridad } \\
\text { de } \\
\text { realización }\end{array}$ & $\begin{array}{l}\text { término } \\
\text { marcado }\end{array}$ & $\begin{array}{l}\text { positivo } \\
\text { grado I }\end{array}$ & $A+1$ \\
\hline ăv + ind. & ăv & $\begin{array}{l}\text { hay toma } \\
\text { de posición } \\
\text { del hablante }\end{array}$ & $\begin{array}{l}\text { seguridad } \\
\text { de } \\
\text { no realización }\end{array}$ & $\begin{array}{l}\text { término } \\
\text { marcado }\end{array}$ & $\begin{array}{l}\text { positivo } \\
\text { grado } 2\end{array}$ & $A+2$ \\
\hline
\end{tabular}

18. En el siguiente cuadro se recogen las combinaciones posibles de periodos condicionales y su frecuencia en los textos analizados:

\begin{tabular}{|c|c|c|c|c|c|c|c|c|c|c|c|}
\hline \multicolumn{12}{|c|}{ Periodos condicionales griegos } \\
\hline \multicolumn{3}{|c|}{ Escala de subjetividad } & \multicolumn{9}{|c|}{ Frecuencia } \\
\hline Nivel & Prótasis & Apódosis & $(T e x t o)$ & Antig. & $\%$ & Tuc. & $\%$ & Prot. & $\%$ & Total & $\%$ \\
\hline 1 & $P+2$ & $A+2$ & & 0 & 0,0 & 0 & 0,0 & 0 & 0,0 & 0 & 0,0 \\
\hline 2 & $P+1$ & $A+2$ & & 0 & 0,0 & 0 & 0,0 & 0 & 0.0 & 0 & 0,0 \\
\hline 3 & $P+2$ & $A+1$ & & 1 & 2,5 & 1 & 5,5 & 2 & 1,2 & 4 & 1,8 \\
\hline 4 & $P+1$ & $A+1$ & & 2 & 5,0 & 1 & 5,5 & 26 & 15,8 & 29 & 13,0 \\
\hline 5 & $P+2$ & A - & & 11 & 27,0 & 3 & 16,5 & 47 & 28,5 & 61 & 27,3 \\
\hline 6 & $P+1$ & A - & & 0 & 0,0 & 3 & 16,5 & 3 & 1,8 & 6 & 2,6 \\
\hline 7 & $\mathbf{P}-$ & $A+2$ & & 3 & 7,5 & 1 & 5,5 & 13 & 8,0 & 17 & 7,6 \\
\hline 8 & $P-$ & $A+1$ & & 2 & 5,0 & 0 & 0,0 & 12 & 7,3 & 14 & 6,2 \\
\hline 9 & $\mathbf{P}-$ & A - & & 22 & 53,0 & 9 & 49,5 & 61 & 37,4 & 92 & 41,2 \\
\hline & & & Totales & 41 & 100,0 & 18 & 100,0 & 164 & 100,0 & 223 & 100,0 \\
\hline
\end{tabular}

19. Como se aprecia en el cuadro hay nueve combinaciones posibles, aunque dos de ellas, niveles uno y dos, se dan sólo en un plano 
teórico, sin realización en la práctica. Puesto que el cuadro señala la mayor o menor presencia de toma de posición del hablante, podría denominarse «escala de subjetividad de los periodos condicionales griegos", siendo el nivel uno el de máxima subjetividad y el nueve el de mínima. Hemos seguido un orden ascendente en la escala, de menor a mayor subjetividad, a la hora de aplicar esta hipótesis a los períodos condicionales registrados.

\section{Aplicación de la hipótesis de trabajo}

20. El número mayor de ejemplos se ha registrado en el nivel nueve. El período condicional está constituido por una prótasis y una apódosis que funcionan como términos no marcados en sus respectivas oposiciones, tienen valor negativo y significan que el hablante no toma posición, es decir, no añade consideración subjetiva alguna, ni al contenido del condicionante ni al enunciado del condicionado. De ahí que resulte un período mínimamente subjetivo. Su representación es $\mathrm{P}-$, A - . La prótasis aparece en indicativo no futuro, la apódosis en indicativo, imperativo, subjuntivo u optativo. Hasta la fecha se ha interpretado que estas apódosis están representadas por expresiones gramaticalmente independientes y expresan el significado propio del modo verbal: objetividad, orden, exhortación y deseo.

21. Los periodos condicionales que aparecen en presente y perfecto de indicativo expresan una idea de simultaneidad, es decir, de actualidad, respecto al acto lingüístico. Por ejemplo:

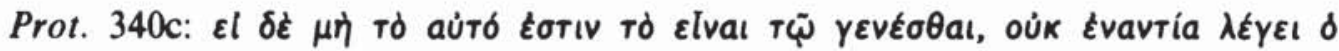

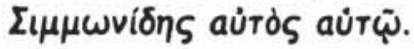

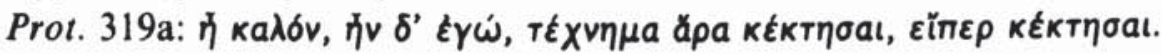

22. Los que tienen su verbo en imperfecto expresan una idea de simultaneidad no actual, en pasado, interpretación que vale para los ejemplos en los que puede aparecer un aoristo, matizando en este caso el aspecto puntual propio de esta forma verbal. Por ejemplo:

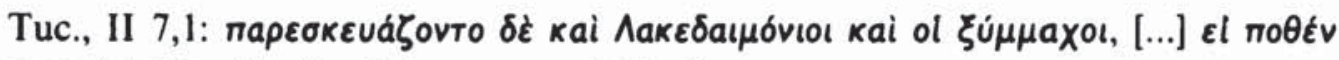

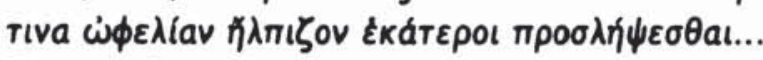

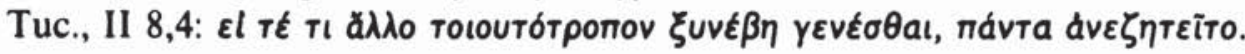

Se da la coincidencia en este caso de que los ejemplos registrados pertenecen a la narración histórica de Tucídides, no habiendo ejemplo 
alguno expresado en los tiempos de pasado del indicativo y que pertenezcan a un tipo de mensaje actualizado.

23. Hay un texto en el que se combina un imperfecto en prótasis con una apódosis en la que el verbo está sobreentendido [żorı], presente de indicativo, por lo que el período condicional ha de ser interpretado como que el contenido del condicionado está situado en un momento posterior al acto lingüístico, pero cuya duración se extiende hasta el presente, momento en el que se sitúa el condicionado:

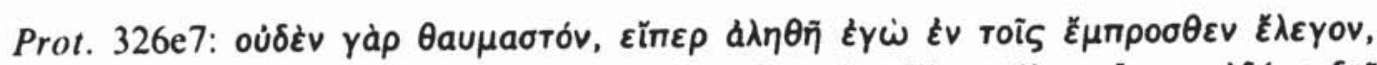

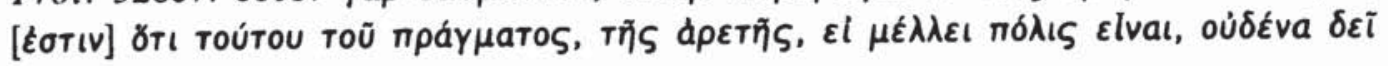

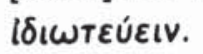

24. Aquellos ejemplos en los que la prótasis está en presente de indicativo y la apódosis en futuro de indicativo, en imperativo, subjuntivo $u$ optativo han de interpretarse como que el hablante enuncia el condicionante sin otra connotación temporal que la simultaneidad respecto al acto lingüístico; en cambio, el condicionado está matizado por el sentido de posterioridad, orden, exhortación o prohibición, y deseo implícito en la expresión verbal. En estos casos el mensaje es directo, se trata de partes dialogadas en las que hay presencia real o imaginaria de un oyente. Veamos un ejemplo de cada combinación:

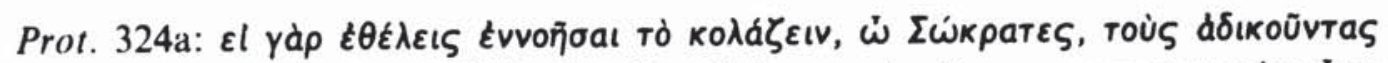

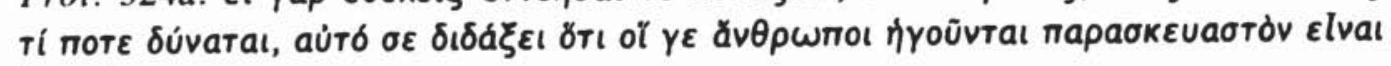

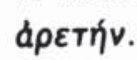

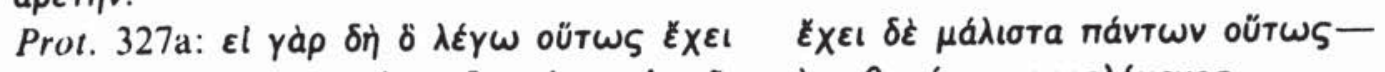

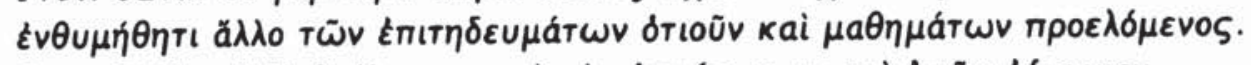

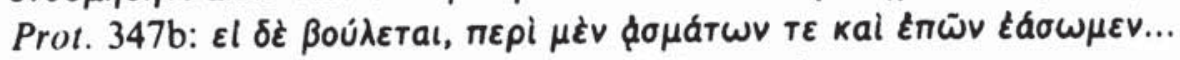

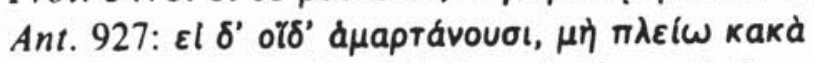

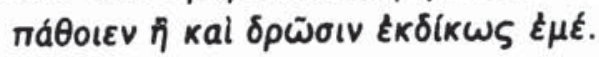

De los períodos condicionales agrupados en este nivel nueve los que expresan simultaneidad actual o alguna relación con el presente pertenecen a fragmentos no narrativos, es decir, no hacen referencia a hechos históricos, sino que corresponden al drama, diálogo filosófico y discurso, en los que el mensaje está actualizado, es directo. En cambio, los que expresan una simultaneidad no actual corresponden a fragmentos histórico-narrativos. Se puede concluir que el rasgo que caracteriza este nivel es la simultaneidad respecto al acto lingüístico, siendo su sentido el de actualidad cuando se trata de un mensaje directo, y el de no actualidad cuando se trata de un mensaje indirecto, histórico-narrativo o referencial. 
25. El esquema representado en este nivel se ha denominado tradicionalmente "periodo condicional real» o "condicional real», entendiendo por tal el hecho de que de darse la suposición, se dará realmente, o seguramente, la consecuencia. Pero como anteriormente se ha afirmado esa denominación resulta inadecuada y errónea ( $(9)$. Por un lado, si por «real» se entiende aquello que se enuncia describiendo algo de la realidad, algo externo al hablante, ese término no vale para denominar el esquema condicional que ahora nos ocupa, pues ha de entenderse que cuando se formula una hipótesis, entendida como el conjunto de prótasis y apódosis, lo que en ella se enuncia es supuesto en cuanto a su realidad: todo acto de suposición consiste en suponer la realidad de lo que se enuncia. Considerar que el sujeto o hablante enuncia la suposición convencido de su cumplimiento, viabilidad o realización, o que duda de esa viabilidad, o que está convencido de su no cumplimiento es, ciertamente, añadir algo más desde su punto de vista en el acto de la suposición. Por consiguiente, todos los tipos de períodos condicionales son enunciados suponiendo la realidad del nexo que une al condicionante con el condicionado; por ello, no es procedente la utilización del término «real» para denominar uno de los tipos posibles. Por otra parte, este esquema, nivel nueve, sólo cabe denominarlo y definirlo de acuerdo con aquellas marcas que lo caracterizan y que son precisamente la ausencia de marcas, es decir, su definición ha de ser negativa, por cuanto que el período está constituido en sus dos miembros por las expresiones no marcadas de sus respectivas oposiciones. La única aportación subjetiva presente en el período condicional es la que consiste en la enunciación del mismo período condicional, que es siempre y por sí mismo un acto subjetivo. De aquí que, al menos en los períodos condicionales, se defina el modo indicativo no como modo de la realidad o de la objetividad, sino como el modo que menos subjetividad expresa.

26. En el nivel ocho de la escala los períodos condicionales están constituidos por prótasis, que funciona como término no marcado y es de valor negativo, y apódosis, que funciona como término marcado con el grado uno en la posesión de la noción básica y es de valor positivo. Su significación consiste en que el hablante no toma posición en el enunciado de la prótasis, pero sí ante el enunciado de la apódosis, en cuanto que manifiesta que él no está seguro de que el período condicional en su conjunto tenga lugar. La prótasis se expresa en indicativo no futuro y la apódosis en optativo con partícula modal ăv. Hemos registrado ejemplos de este nivel en Antígona y Protágoras, no así en el fragmento analizado de Tucídides. Constituye una frecuencia baja $(6,2 \%)$ y 
se representa por $\mathbf{P}-, \mathbf{A}+1$. Los textos analizados corresponden a parte dialogada, ninguno a narración histórica. En la apódosis se registran formas temporales en presente, perfecto, imperfecto y aoristo de indicativo; la apódosis es expresada en optativo con partícula modal, lo que significa que el hablante toma posición añadiendo al contenido del enunciado un dato sobre la seguridad respecto a la realización del período en su conjunto - noción básica-, siendo en este esquema su noseguridad. Destaca en los textos el hecho de que están actualizados todos los períodos condicionales, incluso aquellos cuya prótasis está en imperfecto o aoristo de indicativo; en estos casos el contenido del condicionante se sitúa, en efecto, en un momento anterior al del acto lingüístico, pero su acción se extiende hasta el momento en el que se formula el período condicional, siendo la apódosis la que actualiza el momento de estos períodos. En el caso del período con prótasis en aoristo efectivamente hay una referencia histórica, la de la labor poética de $\mathrm{Si}$ mónides, pero es, como en los casos de imperfecto (Prot. 346d y 349c), un contenido cuya referencia se extiende hasta el momento en el que se formula el período condicional, por lo que adquieren esa valoración de actualidad.

La no-seguridad del hablante en cuanto a que el período condicional tenga lugar es un primer grado en la noción básica; ello no es obstáculo para interpretar que el nexo entre condicionante y condicionado sigue siendo supuesto como "real», al igual que en todos los períodos condicionales. Veamos los ejemplos siguientes:

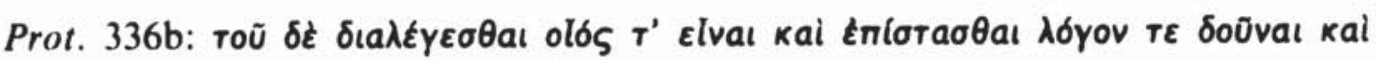

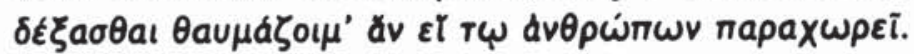

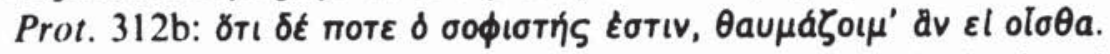

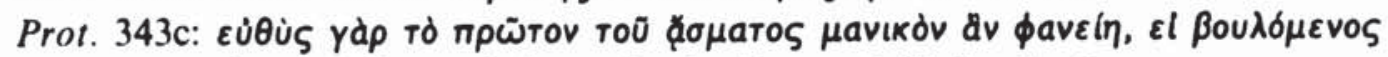

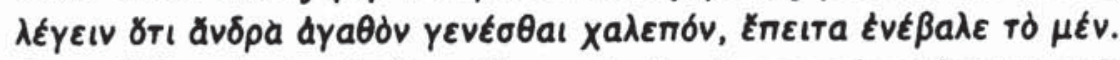

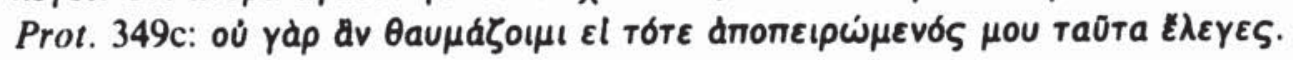

Tradicionalmente este esquema ha sido denominado «condicional mixta real-potencial». En el esquema del nivel nueve ya se ha comentado que el término "real» es inadecuado para denominar los períodos condicionales, incluso los que son definidos como «mixtos», al olvidar que la suposición es enunciada siempre en cuanto a su realidad. Por otro lado, el término "potencial» será comentado a propósito del análisis del nivel cuatro, pero valga desde ahora el mismo comentario: no se trata en un período condicional de que el hablante exprese que, dada una suposición, la consecuencia se puede realizar o no si hay optativo con partícula modal en la apódosis, sino que lo que caracteriza la ex- 
presión en optativo con ăv es la no seguridad del hablante no respecto a la consecuencia, sino respecto al conjunto del período condicional, esto es, no está seguro de que se realice el condicionante ni el condicionado. En el texto Prot. $312 \mathrm{~b}$ el optativo «me sorprendería» significa que el hablante, en esta ocasión Sócrates, no está seguro de que se dé la condición "si tú conoces lo que es el sofista", y tampoco lo está a la vez de que "sé la consecuencia»: su no seguridad estriba en la realización del período completo y no, como hasta ahora se ha interpretado, que supuesta la condición de que conoces lo que es el sofista, yo puedo sorprenderme o no. Entendemos, por el contrario, que en este período, al igual que en todos, la suposición de la condición como "realizada» implica automáticamente la "realización» de la consecuencia. La sorpresa de Sócrates va a existir "realmente», "ciertamente» o "seguramente», con tal de que se dé el condicionante. En este esquema ocurre que a una prótasis no marcada, que, por tanto, sólo enuncia el contenido de la suposición, sigue una apódosis en la que el enunciado de la consecuencia está ampliado con el dato del hablante de que él no está seguro de que el período vaya a tener lugar o no, es decir, de que "conozcas lo que es el sofista y, en consecuencia, me sorprenda". De este modo es preferible entender que este tipo de esquema, denominado mixto por la gramática tradicional, es una posibilidad más de combinación, cuya frecuencia es ciertamente baja, pero que se caracteriza por un mayor grado de subjetividad en la apódosis en relación con la subjetividad existente en el nivel nueve de la escala.

27. En el nivel siete de la escala han sido registrados diecisiete períodos en los tres autores. Se representa por $\mathbf{P}-, \mathbf{A}+2$. La prótasis de este nivel funciona como término no marcado, tiene valor negativo y se expresa, al igual que en los niveles ocho y nueve, en indicativo no futuro. La apódosis funciona como término marcado, presencia de la partícula modal, tiene valor positivo y se expresa en modo indicativo con ăv. Significa que el hablante enuncia el período condicional añadiendo en la apódosis su toma de posición - noción básica-, en cuanto a su seguridad respecto a la realización del periodo en su conjunto. La expresión de la apódosis en indicativo con partícula modal representa un grado más en la posesión de la noción básica por cuanto el hablante no sólo expresa su no-seguridad, sino que además expresa su seguridad de que no tendrá lugar. Es el grado dos del término marcado. Coincide que el momento al que se refiere la suposición es generalmente anterior, por lo que cabría entender que el hablante cuenta ya con una referencia que le permite enunciar la consecuencia sabiendo si el hecho 
de la suposición ha tenido lugar o no. La prótasis se expresa generalmente en imperfecto y aoristo de indicativo, lo que sitúa el contenido del condicionante en un momento anterior al del acto lingüístico. La apódosis aparece en esas mismas formas verbales, lo que indicaría una secuencia de simultaneidad en el pasado entre el contenido del condicionante y el del condicionado. No obstante se dan textos en los que aparece imperfecto con aoristo y viceversa; en estos casos se debe tener en cuenta la categoría aspectual propia de estas formas temporales y el hecho de que algunos verbos carecen de aoristo (p. ej., $\varepsilon l \mu i$ ). Hay un texto (Prot. 353d) en el que aparece un presente de indicativo en la prótasis seguido de imperfecto con äv en la apódosis: en este caso no debemos olvidar que el período condicional depende a su vez de un verbo regente anterior expresado en optativo con ăv. Prueba de que no existe una gran diferencia entre las formas del imperfecto y las del aoristo es el hecho de que se registran textos en los que a una prótasis en imperfecto sigue una apódosis doble, una en imperfecto y otra en aoristo (Prot. 313a, aoristo e imperfecto; 335a, imperfecto y aoristo). Veamos algunos ejemplos:

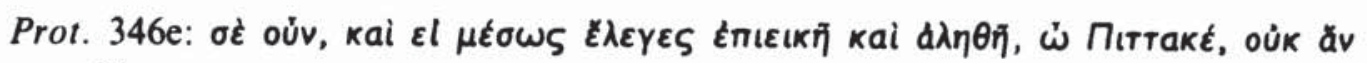

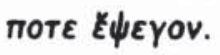

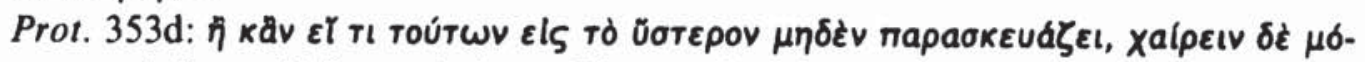

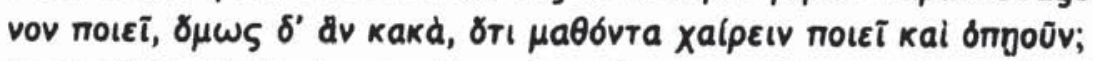

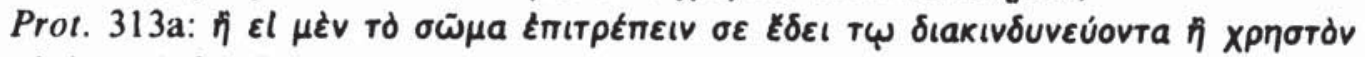

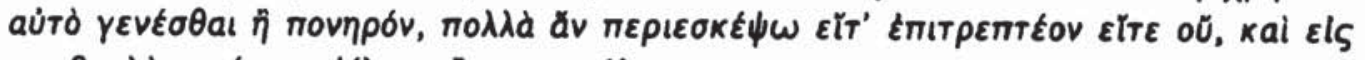

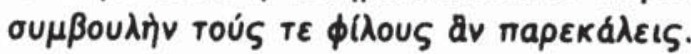

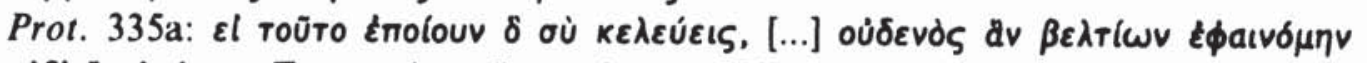

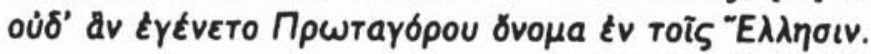

28. Conviene recordar que cualquier traducción a una lengua implica una cierta adulteración de la expresión original, fenómeno que es más evidente cuando se trata de los períodos condicionales, puesto que constituyen un sistema diferenciado en cada lengua. Ello se comprueba en la propia lengua griega, que presenta las variantes más arriba indicadas y que tradicionalmente se han clasificado en cuatro grupos principales y dos o más secundarios; clasificación que difiere de la que, siguiendo los mismos criterios, se ha realizado en latín, lengua en la que no se habla de «eventuales» ni de «iterativas»; por otro lado, la clasificación griega no se puede reproducir ni en castellano ni en francés, pues, en efecto, los sistemas difieren notablemente. Así, suele decirse que la irrealidad se expresa en latín en imperfecto o pluscuamperfecto de subjuntivo, reservando la posibilidad al presente y perfecto; en castellano, 
en cambio, la irrealidad se expresa por medio del pluscuamperfecto de subjuntivo y del llamado potencial compuesto. Sin embargo, se admite en griego la existencia de "irreales de presente» y de «irreales de pasado"; si esto fuera así ¿cómo reproducir en castellano las expresiones griegas correspondientes a esos matices? Consideramos que este problema no ha sido suficientemente analizado y, generalmente, los estudiosos se han limitado a repetir que la irreal de presente se expresa en imperfecto y la de pasado en aoristo. ¿Bastaría en ese caso acompañar la expresión verbal castellana con un adverbio que precise el ahora (presente) y el antes (pasado)? Entendemos que no.

29. Si el imperfecto de indicativo expresa una acción durativa en un momento anterior al acto en el que el hablante expresa su mensaje y, por su parte, el aoristo expresa una idea puntual, generalmente anterior también al acto lingüístico, no cabe hablar de irrealidad de presente, pues el contenido del condicionante tanto en imperfecto como en aoristo refieren algo anterior al acto, por lo que, siendo pasado, no puede ser interpretada esa acción como "[irreal de] presente».

30. Por otro lado, el término «irreal» designa aquello que no sólo no es "real", sino que está "contra lo real" (in-reale), por lo que no puede referirse a aquellos períodos condicionales en los que el nexo entre condicionante y condicionado es supuesto en cuanto a su realidad, pero de los que el hablante está convencido de su no realización. En todo caso lo "real» puede oponerse a lo "no-real», pero hablar de «irrealidad» es introducir otra variante que metodológicamente conduce a un error en el análisis.

31. Así pues, no aceptamos, en primer lugar, la diferencia presente/pasado en las llamadas tradicionalmente «irreales»; en segundo lugar, excluimos el término «irreal» por no ser adecuado a lo que el período condicional en cuestión significa; es decir, no se trata de que el contenido de la suposición sea contrario a la realidad, sino que lo que en este esquema (nivel siete) se señala es que el hablante está seguro de que esa suposición no ha tenido lugar; y su seguridad le viene porque generalmente la referencia temporal del contenido que enuncia es anterior al momento de emitir ese mensaje. En el ejemplo Prot. 346e los imperfectos de prótasis y apódosis se suelen traducir por pretérito imperfecto de subjuntivo y por pluscuamperfecto de subjuntivo o bien por potencial simple o compuesto respectivamente: «si dijeras algo medianamente razonable y verdadero, Pítaco, no te habría reprochado" [o «... no te hubiera reprochado" / "no te reprocharía»], pretendiendo marcar una diferencia temporal que en griego formalmente no existe. En cambio, el 
aoristo en prótasis suele ser traducido por pluscuamperfecto de subjun-

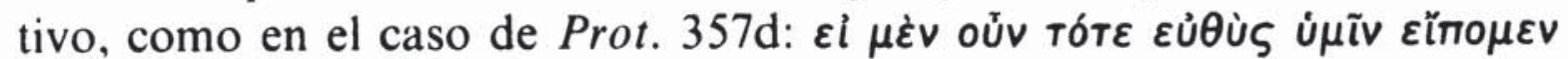

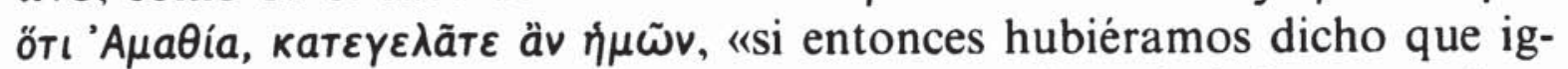
norancia, os hubierais burlado de nosotros». Tal vez habría que replantear la cuestión de la traducción de los períodos condicionales intentando reproducir en castellano el significado que tiene en griego cada período condicional, pues actualmente son también traducidos por pretérito imperfecto de subjuntivo y potencial simple aquellos otros períodos denominados tradicionalmente "posibles".

32. En el nivel seis se han registrado ejemplos en Tucidides y Protágoras. La prótasis funciona como término marcado, - - - del optativo, cuya noción básica consiste en la toma de posición del hablante al enunciar el contenido del condicionante proyectándolo en una secuencia temporal respecto al acto lingüístico; tiene valor positivo. La apódosis funciona como término no marcado, ausencia de partícula modal, su valor es negativo. La proyección temporal de la prótasis es el grado uno del término marcado y significa que su contenido es no necesariamente posterior. Se representa por $\mathrm{P}+1, \mathrm{~A}-$. En conjunto el período condicional significa que dado un condicionante cuyo contenido no es necesariamente posterior al momento en el que se enuncia, el condicionado se limita a enunciar la consecuencia sin ninguna connotación subjetiva. Como en el nivel nueve la apódosis podría aparecer en indicativo, imperativo, subjuntivo y optativo.

$\mathrm{Al}$ analizar los ejemplos de este nivel debemos tener especial precaución con aquellos optativos que aparezcan en lugar de otras formas verbales (optativos oblicuos), ya que no tienen la noción básica asignada al optativo, sino que significan algo ajeno al sentido propio de este modo al entrar en juego una dependencia sintáctica particular; ello aconseja que esos ejemplos con optativo oblicuo sean eliminados de este nivel.

33. La apódosis de los ejemplos registrados aparece en imperfecto y pluscuamperfecto en el caso de Tucídides y en presente de indicativo en el del Protágoras. En ambos textos la presencia del indicativo significa simultaneidad del condicionado con el momento del acto lingüístico: simultaneidad actual, presente de indicativo, en los ejemplos del Protágoras $(329 a, 329 b, 334 b)$, dado que el mensaje está actualizado en el diálogo filosófico, y simultaneidad no actual, imperfecto y pluscuamperfecto, en los ejemplos de Tucídides (II 5,1; II 5,4; II 8,4), en los que el mensaje está relatando algo ya pasado, es un texto histórico, pero que aparece expresado de acuerdo con el momento en el que enuncia el 
período condicional; la diferencia entre pluscuamperfecto e imperfecto es formal, dado que el verbo $\hat{\rho} \omega \dot{v v u \mu \iota}$ en el tema de perfecto medio-pasivo adquiere significación semejante al tema de presente. Veamos algunos ejemplos:

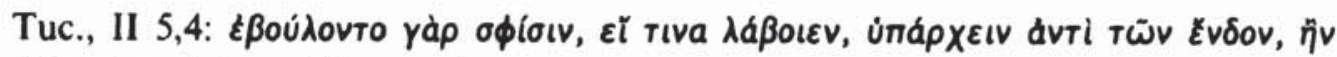

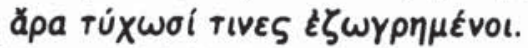

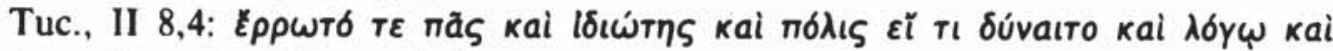

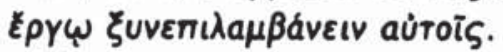

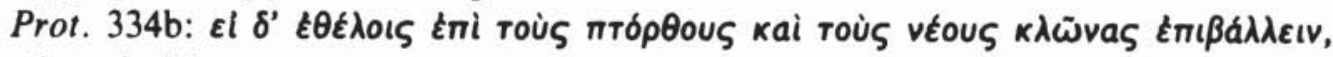

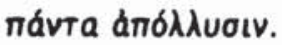

Este esquema es denominado también «mixto potencial-real» por la gramática tradicional, al haber optativo en la prótasis e indicativo en la apódosis, generalmente. En un plano teórico no puede excluirse la presencia posible de los modos indicados en $\S 32$; lo cierto es que en los ejemplos registrados sólo ha aparecido el modo indicativo. Como en los esquemas anteriores la denominación tradicional se considera inadecuada.

34. En el nivel cinco de la escala han sido registrados ejemplos en los tres autores estudiados. La prótasis funciona como término marcado (subjuntivo o futuro de indicativo), valor positivo, su noción básica, toma de posición del hablante, es el grado dos y significa que el condicionante es proyectado hacia un momento necesariamente posterior al acto lingüístico; es decir, no sólo se proyecta hacia un momento no precisado (grado uno), sino que se precisa un poco más al concretar que ese momento es necesariamente posterior (grado dos). La apódosis carece de partícula modal, tiene valor negativo y se puede expresar en indicativo, imperativo, subjuntivo u optativo. La doble posibilidad de expresión de la prótasis en este nivel ha de interpretarse como una doble opción en el plano del significante, sin que ello implique una diferencia sustancial en el plano del significado. En uno y otro caso está claro que el contenido del condicionante puede interpretarse como proyectado hacia un momento posterior. Sin embargo, generalmente se interpreta que la prótasis en subjuntivo, sobre todo cuando va seguida de una apódosis en presente de indicativo, tiene sentido de repetición en el presente. Como antes indicamos $(\S 31)$, es necesario tener en cuenta la peculiaridad del sistema condicional griego y la dificultad de verter con exactitud lo que la lengua griega expresa. Admitiendo este hecho, es posible dar a la prótasis en subjuntivo una significación constante, que no se modifica según la expresión formal del condicionado, sino que ha de interpretarse que el periodo condicional en su conjunto cambia su signifi- 
cación cuando a una misma expresión en la prótasis sigue una apódosis en presente de indicativo, imperativo o futuro de indicativo. No es suficiente decir que el subjuntivo y futuro de indicativo difieren en cuanto al grado de realización concebido por el hablante, esto es, realización segura en indicativo, realización esperada en subjuntivo. Si el único rasgo común entre ambas expresiones es la proyección posterior del contenido en la suposición, no cabe hablar ni de mayor seguridad ni de espera, puesto que en las dos se enuncia una suposición simplemente: «si tú puedes (a partir de este momento)...», «si aparece o no (a partir de ahora)...», "si va a quedar impune...", o "si voy a morir..." son prótasis en las que hay un intervalo de tiempo entre el momento de enunciar el período y el momento en el que se sitúa el contenido supuesto, lo que da el sentido de posterioridad que caracteriza la expresión del condicionante tanto en subjuntivo (que podría ser dudoso para algunos) como en futuro de indicativo.

35. La apódosis, sin marca, enuncia simplemente la consecuencia. Definir períodos distintos («eventuales», «iterativos de presente») por el hecho de que se exprese en futuro de indicativo o imperativo, por un lado, o en presente de indicativo, por otro, es querer resaltar un matiz subjetivo inherente al sentido que tiene el modo verbal usado en la apódosis. Es decir, si en el presente de indicativo sólo se enuncia la consecuencia sin más connotaciones, cuando ésta se expresa en imperativo, subjuntivo, futuro de indicativo u optativo, igualmente se enuncia la consecuencia pura y simple, sólo que en estas formas verbales se da el sentido de orden, prohibición, exhortación, posterioridad o deseo implícito en ellas, pero en ningún caso hay manifestación acerca de la seguridad del hablante respecto a la realización del periodo. Ejemplos con las variantes apuntadas.son:

Tuc., II 34,2: (apódosis en pres. ind.):

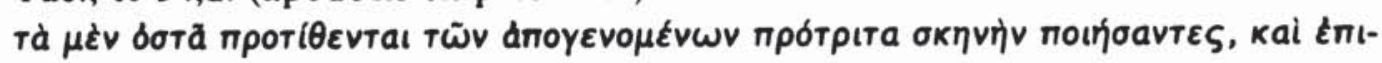

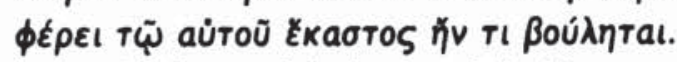

Prot. 328b: (apódosis en pf. ind.):

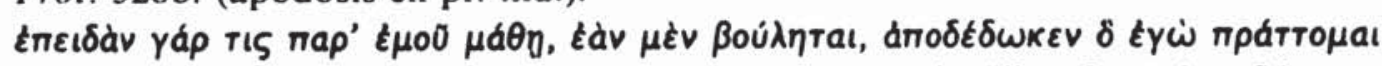

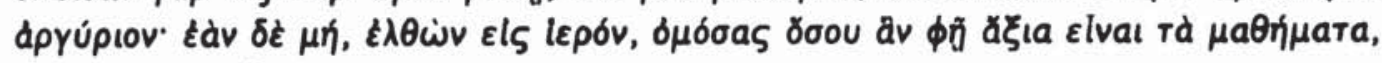

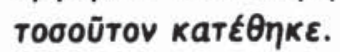

Ant. 461: (prótasis en fut., apódosis en pres. ind.): $\varepsilon l \delta \dot{\varepsilon}$ Toũ Xpóvou

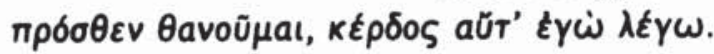

Prot. 355c: (prótasis en subj., apódosis en fut.):

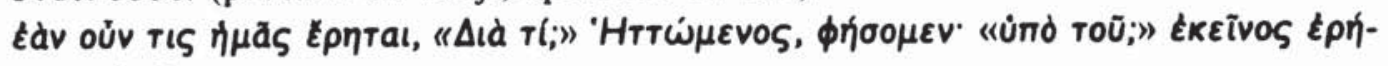

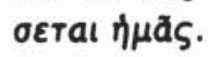


Prot. 348a: (prótasis en subj., apódosis en imperat.):

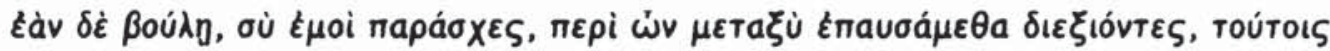

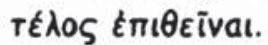

Ant. 306: (prótasis y apódosis en fut. ind.):

$\varepsilon l \mu \eta \dot{~ T o ̉ v ~ a u ̉ T o ́ x \varepsilon i p a ~ T o u ̃ d e ~ t o u ̃ ~ t a ́ \phi o u ~}$

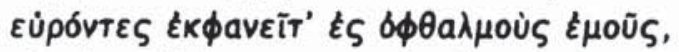

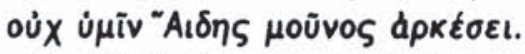

Ant. 58: (prótasis en fut. ind., apódosis en imperat.):

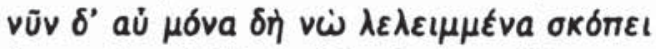

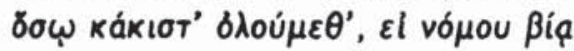

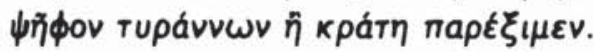

36. Así pues, no admitimos la denominación de «eventuales»e «iterativas de presente» dadas tradicionalmente a los períodos condicionales que responden al esquema representado en el nivel cinco. En efecto, esas definiciones mezclan conceptos dispares como realidad, posibilidad, eventualidad, repetición, que resta coherencia al análisis. Entendemos, por el contrario, que las expresiones de una prótasis en fut. de ind. y en subj. tienen una significación constante, la de proyectar el contenido del condicionante hacia un momento necesariamente posterior al acto lingüístico. Igualmente entendemos que una apódosis sin la partícula modal no modifica el sentido de la prótasis, sino que enuncia el condicionado sin añadir matiz alguno del hablante sobre su seguridad en cuanto a la realización del periodo. Conviene recordar además que sólo en la lengua griega se reconoce una significación específica para la expresión de prótasis en subjuntivo, la de la «eventualidad»; en otras lenguas como la latina ese matiz no existe o está inmerso en las que denominan «posibles».

37. En el nivel cuatro se han registrado ejemplos en los tres autores. En los dos elementos oracionales aparece el optativo. La prótasis funciona como término marcado (-เ- del optativo) que, como en el nivel seis, posee la noción básica en el grado uno y significa que el hablante toma posición al proyectar el contenido del condicionante hacia un momento no necesariamente posterior. La apódosis, también en optativo, posee la noción básica en grado uno y significa que el hablante toma posición al enunciar la consecuencia añadiendo que él no está seguro de la realización del período. El momento no necesariamente posterior puede ser entendido como un momento simultáneo o posterior al acto lingüístico. En el texto puede haber algún adverbio que precise ese momento, pero por sí misma la expresión en optativo no lo concreta. Es definido habitualmente este esquema como "posible» o "potencial», en cuanto que si se da la condición, el condicionado se puede dar o no; en otras palabras, no es seguro que se dé la consecuencia. 
38. Desde nuestro punto de vista el análisis tradicional mezcla dos conceptos heterogéneos, realidad y posibilidad. Al mismo tiempo, lo que define el tipo de período condicional es la forma verbal de la apódosis (optativo con partícula modal: posibilidad), mientras que cuando el esquema lleva en la apódosis un imperfecto de indicativo se define como «iterativa de pasado". En primer lugar, "realidad» se opone a "no-realidad" ${ }^{7}$. Lo posible es otro concepto que no participa de la realidad, por cuanto que lo que es posible, en cuanto tal, no es real, es decir, lo posible es no-real. Por consiguiente, hablar de "posible" es olvidar que un periodo condicional, desde que es enunciado, es supuesto como real, nunca como posible. Cuando en la apódosis de este nivel el hablante toma posición añadiendo su no seguridad respecto a la realización del período condicional en su conjunto, se sigue empleando el mismo criterio en el análisis, sólo que, a diferencia de los períodos condicionales del nivel nueve, el hablante añade una consideración subjetiva al enunciado de la hipótesis.

39. En segundo lugar, la no seguridad del hablante respecto a la realización del período condicional puede estar determinada por una falta de referencia temporal, dado que el contenido de la suposición no remite necesariamente a un momento anterior. La interpretación de este esquema dista, pues, de la dada tradicionalmente, manteniendo una coherencia y homogeneidad en el análisis y en los conceptos. Sirvan los siguientes ejemplos:

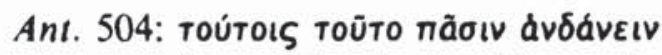

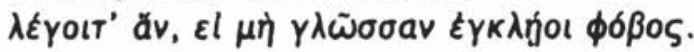

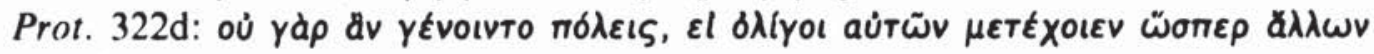

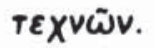

40. En el nivel tres se han registrado cuatro ejemplos. La prótasis funciona como término marcado, en subjuntivo o futuro de indicativo, tiene valor positivo y posee la noción básica en grado dos, es decir, su significación consiste en que el contenido del condicionante no sólo se proyecta en el tiempo, sino que esta proyección ha de ser necesariamente posterior al momento del acto lingüístico. La apódosis funciona también como término marcado, se expresa en optativo con partícula modal, tiene valor positivo y su noción básica es poseída en grado uno; significa que el hablante toma posición en el enunciado del condicionado añadiendo su no seguridad en cuanto a la realización del periodo

\footnotetext{
${ }^{7}$ H. Vairel, art. cit., $\S 24$.
} 
condicional en su conjunto. El contenido de la hipótesis no es necesariamente anterior. Se representa por $\mathrm{P}+2, \mathrm{~A}+1$. La gramática tradicional define este período como «mixto eventual-potencial». Como antes se señaló, desde un punto de vista lingüístico tal definición es contradictoria e inadecuada. Se trata de un contenido supuesto como real cuyo condicionante se proyecta hacia un momento posterior al acto lingüístico, seguido de una consecuencia en la que el hablante añade su apreciación de no estar seguro de que tal período condicional tenga lugar. Algunos ejemplos son los siguientes:

Ant. 1169:

$\varepsilon a d v \delta^{\prime} d n \tilde{n}$

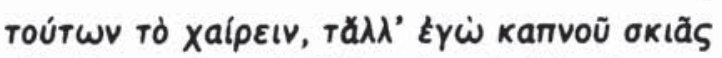

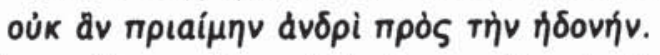

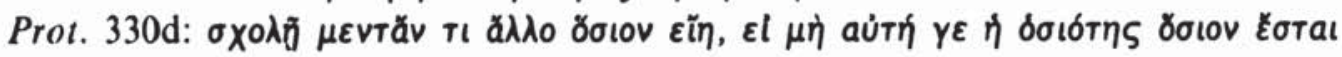

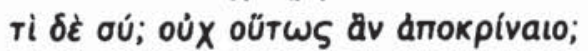

La expresión de la apódosis en el primer ejemplo marca la no seguridad de que ese período se dé en la realidad; pero de darse la suposición es seguro que «no compraría nada a cambio de la alegría».

41. En los niveles uno y dos no han sido registrados ejemplos en ninguno de los tres textos analizados. Por tanto, sólo cabe hacer aquí un planteamiento teórico de unas casillas previstas en el plano de la lengua, pero no realizadas en el plano del habla. Así el nivel dos tendría una prótasis con función de término marcado, valor positivo en grado uno, expresada en optativo y significaría que el hablante proyecta el contenido de la suposición hacia un momento no necesariamente posterior. La apódosis funcionaría como término marcado, con valor positivo en grado dos, expresada en indicativo con partícula modal y significaría que el hablante toma posición al añadir al enunciado de la consecuencia que él no sólo no está seguro acerca de la realización del período condicional (grado uno), sino que además está seguro de su no realización (grado dos).

42. El nivel uno está formado por una prótasis y una apódosis que funcionarían, caso de registrarse, como términos marcados con valor positivo en grado dos, expresadas en subjuntivo o futuro de indicativo el condicionante, en indicativo con partícula modal el condicionado; significaría que al contenido de la prótasis proyectado hacia un momento necesariamente posterior al acto lingüístico seguiría un condicionado a cuyo contenido el hablante añadiría su seguridad de que tal período condicional no ha tenido lugar. 
43. La máxima subjetividad que se registra en estos dos niveles explica su no registro en el plano del habla. En efecto, cuando en la prótasis el hablante proyecta el enunciado de la suposición hacia un momento no necesariamente posterior (grado uno, nivel dos) o necesariamente posterior (grado dos, nivel uno) y la consecuencia incluida en la apódosis expresa la seguridad del hablante de que el período condicional no se ha realizado (ni se realizará) por su referencia de pasado, revela una contradicción que convierte los dos niveles mencionados en esquemas posibles, pero ilógicos.

\section{Conclusiones}

44. La causa que justifica un nuevo análisis de los períodos condicionales griegos es la insuficiencia de las definiciones y clasificaciones dadas hasta ahora, bien porque en ellas se ha considerado sólo una parte, condicionante o condicionado, bien porque se ha aplicado un criterio inadecuado, sea el de realización, erróneo desde el principo, sea el de extensión, que se interesa sólo por el plano semántico, ya sea mezclando varios criterios de los que resulta una visión heterogénea, ya sea aplicando puntos de vista diferentes según convenía al objeto de estudio.

45. Se entiende que los errores anteriores pueden ser evitados limitando el análisis del objeto a una consideración estrictamente lingüística.

46. El período condicional ha de ser estudiado como un conjunto de dos elementos oracionales llamados condicionante, subordinado o prótasis el primero, y condicionado, principal o apódosis el segundo. Aquél enuncia un supuesto, éste su consecuencia.

47. Frente a las teorías tradicionales entendemos que el enunciado del elemento condicionante consiste en suponer la realidad de aquello que se enuncia y el vínculo del condicionado con el condicionante es supuesto igualmente como real y necesario.

48. Todo período condicional es en sí mismo un primer acto subjetivo, el que consiste en suponer la relación condicionante-condicionado. Puede haber una segunda aportación subjetiva del hablante que se añade a la prótasis, a la apódosis, o a las dos al mismo tiempo.

49. El estudio realizado tiene un enfoque estructural: examinadas las formas posibles en cada uno de los dos elementos de un período, se 
establece en cada elemento oracional un sistema de oposición, en el que cada expresión se define por estar caracterizada morfológicamente o no; la caracterización en el plano del significante implica la posesión de una noción básica en el plano del significado, noción básica que añade un dato más en la significación de la expresión, del que carece la expresión no caracterizada. De este modo se obtiene una expresión que funciona como término marcado con valor positivo y otra que funciona como término no marcado con valor negativo. Dado que en nuestro análisis el término marcado presenta dos expresiones distintas pero no opuestas, hemos de entenderlas como que ambas poseen la noción básica implícita en la marca, pero en un grado distinto la una respecto a la otra: grado uno (proyección no necesariamente posterior en prótasis, no seguridad en apódosis) y grado dos (proyección necesariamente posterior en prótasis, seguridad de no realización en apódosis).

50. En un sistema de oposición lingüística no es obstáculo la presencia de dos grados en la posesión de la noción básica. En efecto, en la prótasis de un período condicional una expresión dada puede proyectar su contenido hacia un momento anterior, simultáneo o posterior en relación con el momento en el que se formula el enunciado del período condicional (término marcado), o no proyectarlo (término no marcado). Ahora bien, la expresión marcada puede precisar ese momento o no. Cuanto más definido esté ese momento, tanto más caracterizada estará la expresión; pero una y otra expresión, con precisión del momento o sin ella, estarán caracterizadas, porque llevan una marca (optativo/subjuntivo-futuro de indicativo) indicadora de la noción básica, que consiste en la toma de posición del hablante. La diferencia estará en la forma de poseer esa noción: un primer grado, expresado en optativo, proyecta el contenido del condicionante hacia un momento no necesariamente posterior; un segundo grado, expresado en subjuntivo y futuro de indicativo, proyecta el contenido del condicionante hacia un momento necesariamente posterior.

51. Por otro lado, en la apódosis de un período condicional una expresión dada puede indicar si el hablante toma posición ante el enunciado de la consecuencia (posee noción básica, término marcado) o no (sin noción básica, término no marcado). La expresión marcada añade al enunciado de la consecuencia un grado de seguridad del hablante sobre la realización o no del período, dato que no aparece en la expresión no marcada. Cuanto mayor sea el grado de seguridad, más caracterizada estará la expresión. La marca portadora de la noción básica es la partícula modal ăv. Cuando la expresión va en optativo con partícula el 
grado es menos preciso, grado uno, y significa que el hablante no está seguro de que el período condicional tenga lugar; la expresión en indicativo con partícula modal expresa un grado más preciso, pues significa que el hablante no sólo no está seguro de si se realizará el período, sino que está seguro de que no va a tener lugar. En este segundo caso, el grado mayor de seguridad viene determinado porque la referencia temporal del contenido del periodo es anterior al acto lingüístico.

52. La referencia temporal que cada elemento oracional indica se concreta en un grado mayor, con respecto al acto lingüístico que consiste en enunciar un período condicional: es posterioridad en la prótasis, $y$ anterioridad en la apódosis. Esta situación contraria en la línea del tiempo puede explicar que no se registren ejemplos en algunos niveles (uno y dos), que siendo posibles en la teoría, no han encontrado realización en la práctica; su contenido contradictorio los excluye.

53. Analizadas y clasificadas las expresiones que aparecen en un período condicional, establecida la noción básica (toma de posición del hablante) que caracteriza a las que tienen una marca formal diferenciadora, expuestos los términos marcados y no marcados, los valores positivos y negativos y la significación de cada una de esas expresiones, se llega a la conclusión de que en griego es posible establecer nueve tipos de períodos condicionales en un plano teórico, de los que sólo se realizan siete en un plano práctico, y que esquematizamos en una escala de subjetividad.

54. Respecto a la frecuencia de ejemplos registrados en los niveles de la escala se puede apuntar los datos siguientes:

a) En los niveles nueve, seis y cinco hay expresiones de la apódosis (imperativo, subjuntivo y futuro de indicativo) que requieren la presencia real o imaginaria de un oyente. Esta presencia se da en aquellos textos que son parte dialogada o discurso, pero no en los que son narración histórica.

b) En los niveles siete y ocho no se han registrado ejemplos en el texto analizado de Tucídides. Su explicación puede estar en el hecho de que al ser una narración histórica el autor (hablante) está situado en una referencia temporal posterior a los hechos narrados, lo que no facilita períodos condicionales de esos dos niveles.

c) Coincide que los períodos condicionales cuya apódosis es $\mathrm{A}+1$ y tienen una prótasis del tipo $P+1$ y $P+2$ sólo se dan en parte de discurso. No se ha registrado ninguno en parte narrativa, al menos en el fragmento analizado. 
d) La frecuencia elevada en los niveles de menor subjetividad, nueve, cinco y cuatro, se puede deber al interés del hablante en expresar el enunciado ante el oyente lo menos subjetivamente posible y, por consiguiente, con una apariencia más universal.

e) En los niveles uno y dos, de máxima subjetividad, no se han registrado ejemplos, siendo posiblemente la causa el carácter ilógico y contradictorio que resultaría de la combinación de una prótasis con contenido posterior y una apódosis de referencia al pasado.

f) Por consiguiente, aunque son nueve los niveles posibles en los que se agrupan las combinaciones de prótasis y apódosis, sólo siete tienen realización en el plano del habla.

55. Entendemos que por primera vez se ofrece un estudio de los períodos condicionales griegos enfocado con un criterio único, coherente en el método y en el análisis, atribuyendo a cada expresión formal una función, un valor y una significación constantes. De acuerdo con ese criterio son clasificados los elementos oracionales y sus posibles combinaciones.

56. Naturalmente las conclusiones de este estudio están referidas a los textos analizados (Antigona, Protágoras y Tuc., II 1 - II 43), comprendidos dentro de un marco temporal situado entre los siglos $\mathrm{v}$ y IV a.C. y en un contexto dialectal fundamentalmente ático. No obstante, esperamos extender la aplicación de este análisis a otros textos, épocas y dialectos, dado que con los ejemplos analizados no se recogen todas las variantes posibles y no podemos excluir de forma definitiva que no haya textos en los que los esquemas de los niveles uno y dos estén presentes o que alguno de los períodos condicionales no registrados en un autor no pueda ser registrado en otra obra o en otro autor en ese mismo tipo de comunicación.

57. Nuestro estudio se ha situado dentro de unos límites estrictamente lingüísticos, porque entendemos que la Lingüística ha de ser considerada como una ciencia específica, diferenciada de la Lógica formal, de la Sociología o de la Filosofia.

\section{Luis Miguel Pino Campos}

